\title{
A DESIGN RATIONALE ANALYSIS METHOD TOWARDS ROBUST ARTIFACT DESIGN
}

\author{
Tsutsui, Yusuke (1); \\ Mitake, Yuya (1); \\ Sholihah, Mar'atus (1); \\ Hosono, Shigeru (2); \\ Shimomura, Yoshiki (1) \\ 1: Department of System Design, Tokyo Metropolitan University; \\ 2: School of Computer Science, Tokyo University of Technology
}

\section{ABSTRACT}

To design a more robust artifact, an artifact design based on a design rationale analysis is pivotal. Errors in previous design rationales that led to the degradation of artifact robustness in the past provide valuable knowledge towards improving the robust design. However, methods for exposing and analysing errors in design rationale remain unclear. This paper proposes a structured method for a design rationale analysis based on logical structuring. This method provides a well-constructed means of identifying and analysing errors in design rationale from the perspective of knowledge operation.

Keywords: Design management, Design process, Decision making, Design cognition, Design rationale

\section{Contact:}

Tsutsui, Yusuke

Tokyo Metropolitan University

Department of Mechanical System Engineering, Faculty of System Design

Japan

tsutsui-yusuke@ed.tmu.ac.jp 


\section{INTRODUCTION}

The amount of information that engineering designers need to consider has been increased because of the increasing complexity of artifact, the diversification of customer needs and the social demand for environmental consideration. As a result, engineering designers have a much wider range of tasks and areas to operate in (Pokojski et al., 2019). On the other hand, the time that the engineers can use to design is limited and, moreover, their knowledge is not perfect. For example, designers seldom know all the criteria and they never have enough knowledge (Ullman, 2001). Additionally, engineering designers cannot retain all the information they need to solve complex design problems in their heads (Bracewell et al., 2009). Thus, designers are compelled to make tentative decisions with bounded rationality. These cognitive limitations of the designer decrease customer satisfaction and cause serious and sometimes fatal accidents as well. Therefore, how to support designers beyond these cognitive limitations is one of the key issues that the engineering design field needs to address.

Knowledge management techniques for design are valuable because the ability of designers to retrieve and use information during the design process has a significant impact on the outcome. Especially, the study of representing, capturing and retrieving knowledge related to decision making in the design process has been studied under the term of design rationale (Wang et al., 2012). By capturing the design rationale, it is expected to support redesign, reuse, maintenance, learning, documentation, collaboration and management of project (Lee, 1997). In order to achieve robust decision making in engineering design, it is necessary not only to manage the design rationale for successful cases, but also to analyse the design rationale for failed cases and generate knowledge from the findings. However, analysis of the errors in the design rationale has not yet been fully explored.

Based on the aforementioned backgrounds, this study constructs a method to analyse the errors in design rationale of individual designer. Specifically, this study constructs a mental model of the designer, which describes the rational reasoning of human under the bounded cognitive resources, and organise its logical structure to provide a perspective for capturing the design rationale retrospectively. Then, this paper arranges a typology of design rationale errors, which provides a perspective for identify the errors of the captured design rationale. By identifying the design rationale errors using the proposed method, useful knowledge can be generated from failed cases, thereby contributing to improve the robustness of future artifact design.

The rest of this paper is structured as follows. In Section 2, related studies are reviewed. Section 3 clarifies the research gap and explains approach. Section 4 provides a detailed explanation of the method used to identify errors in design rationale. In Section 5, the proposed method is applied to one of the failed cases. Lastly, this paper culminates in Section 6.

\section{LITERATURE REVIEW}

\subsection{Decision making in design process}

Decision making is the process of making a decision characterised by identifying alternatives, comparing alternatives to criteria so-called evaluation, and finally deciding on a resolution. Meanwhile, a decision itself is the decision-maker commitment to perform a certain activity by using specific resources (Ullman, 2001). In the context of developing a new artifact, the decision making activities during the design process are complex. This due to not only the designer is targeted to satisfy customers' needs and values but also to ensure the proper profit generation for the company. Any decisions made have a crucial impact on the design solution, the business, and the design process (Hansen and Andreasen, 2004). Hansen and Andreasen (2000) have conceptualized the design decision making through the decision node model (Hansen and Andreasen, 2000). This model presents an elementary decision making activity in the design process involving six sub-activities. This decision making activity starts from (1) to specify design solution alternatives, then (2) to evaluate those alternatives, (3) to validate a design solution, (4) to navigate through the solution/activity space, (5) to unify the current decision into consistent wholes and (6) to make a decision. Moreover, this model articulates that the object synthesised during a product development project includes three artifact elements i.e., the product, the life phase (such as production and distribution systems), and the meetings among the product, the operator, and the life phase system. Therefore, the object of any decision making during the design process involves characteristics and has consequences related to these artefact elements (Hansen and Andreasen, 2004). 
Despite the available guidelines for decision making in design methodology literature, an empirical study of engineering designers has shown that design methods are sparsely adopted in industrial practice (Hansen and Andreasen, 2004). The underlying differences between theory and practice of design decision making include the fact that the engineering designers regard decision making as a complex collection of interwoven decisions and clarifications. A design decision is a tentative decision made based on the currently available information. This tentative decision is then considered verified if new criteria and clarification emerge to the point of satisfactory support the tentative decision. Thus, the decision object is neither constant nor static. It evolves as the results of clarifications and possibilities following different ranges and boundaries during the design process.

\subsection{Design rationale in the field of knowledge management for engineering design}

In the field of knowledge management for engineering design, the way to capture the design rationale is discussed in order to develop the design knowledge management system, which supports the designers' decision making under the engineering design problem. Design rationale is an explanation of why an artifact, or some part of an artifact, is designed the way it is (Lee and Lai, 1991) and regarded as the beneficial knowledge for complexity management of design process, effective maintenance, reverse engineering, design reuse and design tractability (Arora et al., 1992). Thus, how to capture the document of design rationale is one of the pivotal issues. In this field, the designer is seen as the engineering professionals that they have the capability of applying technical knowledge, making decisions, and adopting courses of action, to solve design problems (Wang et al., 2012) .

Research into capturing and mapping the rationale for complex decisions originated with the Issuebased information system (IBIS) in 1970 (Kunz and Rittel, 1970), and a variety of systems derived from IBIS have been developed. Garcia (1992) mentioned there three major models for representing design rationale: argumentation-based design rationale, action-based design rationale, and modelbased design rationale (Garcia and Howard, 1992). The first approach represents the design rationale as a set of argument (pros and cons) nodes and decision nodes attached to issue nodes. IBIS is categorised under this approach. The Pathogen-Host Interaction Data Integration and Analysis System (PHIDIAS) is another example of the design rationale management system based on this approach (Shipman and McCall, 1997). In the action-based approach, the design rationale the all information and action during the design. Based on this approach, the design rationale can be automatically recorded as a designer's activity log during the design process, exemplified by Lakin's electronic notebook project (Lakin et al., 1989). At last, the model-based approach claims the design rationale should be contained the domain specific knowledge such as functions, behavior of artefacts and design principles. Advanced Design Coordination Tools (ADCT), developed by Domeshek and holman (2002), is the on-line web-based environment based on this approach (Domeshek and Holman, 2002).

\section{RESEARCH GAP AND APPROACH}

\subsection{Research gap}

As explained in 2.2, how to capture and reuse the decision rationale has been discussed in the field of engineering design so far. On the other hand, not only capturing design rationales in successful cases, but also capturing and reusing design rationales in failed cases may enable the more robust decision making in design. However, the current research lacks the method to analyse the error behind design rationale. Thus, it is still not possible to reuse the knowledge that would be captured from failed cases.

\subsection{Approach}

This study defines design rationale as "premise information, such as beliefs and knowledge, referred to reasoning in the designer's decision making, and the results of reasoning including design alternatives." The designer is regarded as an agent who repeatedly makes tentative decisions based on a certain amount of rational reasoning, even when cognitive resources are limited. Furthermore, this paper assumes that errors in the design rationale are caused by the limitations of the designer's cognitive resources.

Based on the above assumptions, this study constructs a mental model of a designer who makes rational decision making under bounded cognitive resources. The foundation of this mental model is constructed by referring to the theory of intention (Bratman et al., 1988), a philosophical theory that presents a model of a rational actor, and then corresponding the logic of reasoning in decision making. 
This makes it possible to retrospectively grasp the rationality under which the designer made decisions with limited cognitive resources, namely, what premises and what logic the designer reasoned based on in decision making. In addition, the model provides a typology of errors that can be included in the designer's decision making. By reconsidering the designer's decision making based on these typologies, it enables analysts to identify errors in the design rationale.

\subsubsection{Planning theory of intention}

Bratman argues that it is necessary to analyse the rationale for coordinating planning and decision making among the actors beyond the constrains of bounded resources and time (Bratman et al., 1988).

He proposed a theory to explain how rational action is derived under bounded cognitive resources and time. According to his theory, human beings reason in advance at an abstract level about the mental states that will drive actions in the future, and as time passes and they acquire information, they reason about concrete mental states and finally practice actions (Bratman et al., 1988). Bratman called the mental state that drives such actions intentions, and the collection of intentions accumulated in the subject through repeated inference of intentions is called a plan(Bratman et al., 1988). Namely, humans reason abstract intentions about actions, i.e., partial plans, in advance, and then reason concrete plans as time passes, leading to actions. In this way, reasoning the intentions of future actions in advance enables rational reasoning that could not be achieved only in the limited time immediately before the action. In addition, the strategy of reasoning about future actions from highly abstract intentions allows humans to make acceptable decisions even in a future world that is difficult to predict.

As explained on the above, the core concept of Bratman's theory is intention, which is defined as a proactive attitude driving future actions and formed based on deliberation. Once the actor forms the intention, it is kept for a period of time in the actor's mind. Moreover, intention drives actions for achieving his/her goal through a means-end reasoning process that searches for and deliberates means. Intention allows rational actors to make future-oriented decisions beyond the bounded resources.

\subsubsection{Logical form in cognitive design process}

Takeda et al. (1990) propose a cognitive model of the design process with logical formalisation to create intelligent computer-aided design systems (Takeda et al., 1990). According to this model, the designer constructs candidates to solve a problem using various types of design knowledge. Then, he/she evaluates whether candidates can solve the problem or not in various ways. This design process is conducted cyclically through refining candidates until deciding which one to hire. This cognitive model is formalised by the following logical framework: $D s U K o \vdash P$. Where $D s$ is a set of logical formulas describing a design candidate, $K o$ is knowledge of object properties and behavior, and $P$ are properties of the design candidate. According to this model, the designer iteratively uses a combination of abduction and deduction to refine and conclude their final design solution. While the designer tries to find a feasible candidate, he/she obtains $D s$ from $P$ and $K o$; this can be regarded as an abduction process. Both the candidate development and evaluation processes can be classified as forms of deduction. In these processes, the designer applies his/her knowledge to refining the candidates.

\section{THE MODEL FOR ANALYSING DESIGN RATIONALES}

\subsection{The mental model of a designer with bounded rationality}

Based on the theories presented in 2.2, this paper proposes the mental model of a designer (Figure 1). This model was constructed based on Bratman's theory explained in 3.2.1 while each module was visualised and named by authors. This model is composed of three segments: mind, body, and surroundings. The analyst cannot observe the designer's mind directly, but can infer it through his surroundings and behavior by presupposing the functions and structures of his mind. Mind and surroundings interact with each other through perceptions and actions that are functions of the body. Mind consists of two mechanisms, i.e., a decision making mechanism and a decision premise mechanism. The decision making mechanism works to select an action that will achieve a goal, and the decision premise mechanism works to operate the mental states that are the reason for decision making. Decision premise is a term used by Simon in the context of organisational theory research (Simon, 1947). Mind includes three component types: the mental state, the operating module, and the reasoning module. The mental state (directed links) is the component that represents the basic condition of the 
mind. The operating module (grey rectangular nodes) is the functional module that adjusts the consistency of mental states, including storage and output. The reasoning module is a functional module as well as an operating module, but its function is to execute reasoning based on input from the mental states. The significant difference between the operating module and the reasoning module is the capacity to store these mental states. The input-output relationships that exist among these three components are a mental state, operating module, and reasoning module, illustrated as directed links in Figure 1.

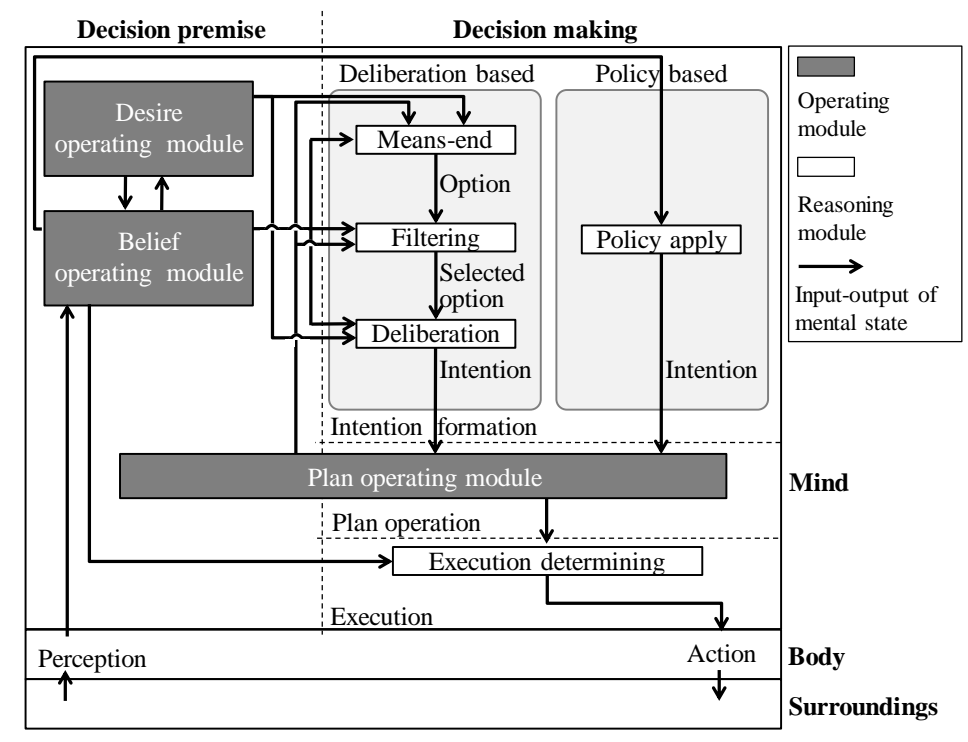

Figure 1. The mental model of a rational actor beyond the bounded cognitive resources

\subsubsection{Decision making mechanism}

In the decision making mechanism, a designer makes a design-related decision to take action. This decision making process has three phases: intention formation, operation planning, and execution. In Figure 1, these phases are presented as three segments separated by a horizontal dotted line.

Intention formation includes two types of processes: a deliberation-based process and a policy-based process, depicted in Figure 1 as the grey background area. In the deliberation-based process, an intention is derived through three reasoning modules: the 'means-end reasoning module', the 'filtering module', and the 'deliberation module'. In the policy-based process, an intention is derived through one reasoning module - the 'policy apply reasoning module'. When solving a problem which has not experienced, an intention is formed through a deliberation-based process. In contrast, when solving a problem which has experienced before, an intention is formed through a policy-based process. In this way, policy-based process, which forms a particular intention in a situation that has been experienced, enables subjects to make efficient use of their limited time. The plan operation is conducted in the plan operating module. The plan operating module has a function to adjust the order of intentions to the order of execution and to adjust the consistency of the intentions. Intentions are executed in the 'execution determining module', which is a reasoning module. This module includes functions that call for intentions that are under the control of the plan operating module, to determine the execution of intentions based on criteria, and to output actions.

\subsubsection{Decision premise mechanism}

A designer stores and operates their mental states, which comprise the decision premise of decision making in the decision premise mechanism. This decision premise mechanism is composed of a desire operating module, belief operating module, and plan operating module.

The functions of the desire operating module are to adjust the desires of the designer based on beliefs inputs and on output desires. The functions of the belief operating module are to adjust the beliefs including knowledge and cognitions of the designer based on the inputs: desire, and perceptions of surroundings. In addition, this module produces outputs such as knowledge and cognitions. The plan operating module has functions to adjust the consistency of intentions, to store intentions, to output intentions, which function as the filter in the 'filtering reasoning module', and to output the intentions of the 'means-end reasoning module'. 


\subsection{Logic of designer's reasoning in decision making}

\subsubsection{A form of logical reasoning in deliberation-based decision making}

The mental state used in each reasoning module is symbolised, as shown in Table 1. Table 2 presents the logical formulas that suggest a form of logical reasoning in deliberation-based decision making. For example, the second line in Table 2 shows the logical structure of the 'means-end reasoning module'. In the means-end reasoning module, ' $O p$ ' which satisfies ' $\mathrm{Km} \cup \mathrm{Op} \vdash \mathrm{Ob}$ ' and ' $\mathrm{Km} \cup \mathrm{Op}$ is consistent' is obtained from $\mathrm{Km}$ and $\mathrm{Ob}$. Namely, the means-end reasoning module functions to drive abduction. On the other hand, in the 'filtering module' and the 'execution determining module', reasoning is performed through deduction. In the filtering module especially, $O p$, which comes from the means-end reasoning module, is rejected only when $\sim O p$ is obtained from $K f$ and $C p$. The deliberation module has a function to consider the most rational option for action from the selected options derived from the filtering module.

Table 1. Mental state symbols in deliberation-based decision making

\begin{tabular}{cl}
\hline Symbols of the mental state & \multicolumn{1}{c}{ Explanation } \\
\hline$O b$ & The design objective \\
$K m$ & Knowledge of means to achieve the design objective \\
$O p$ & Options of the design operation \\
$C p$ & Cognitions of the design object's properties \\
$K f$ & Knowledge to filter the unfeasible options \\
$I$ & Intention, Proactive attitude driving future actions \\
$C t$ & Triggers required to execute the intention \\
$K t$ & Knowledge of triggers required to execute the intention \\
$A$ & Action related to the design, design operation \\
\hline
\end{tabular}

Table 2. A form of logical reasoning in deliberation-based decision making

\begin{tabular}{ccc}
\hline Reasoning module & Condition & A form of logical reasoning \\
\hline Means-end reasoning module & $K m \cup O p \vdash O b$ & Abduction to obtain $O p$ from $K m$ and $O b$ \\
& $K m \cup O p$ is consistent & \\
Filtering module & $K f \cup C p \vdash \sim O p$ & Deduction to obtain $O p$ from $K f$ and $C p$ \\
Execution determining module & $K t \cup I \cup C t \vdash A$ & Deduction to obtain $A$ from $I, C t$ and $K t$ \\
\hline
\end{tabular}

\subsubsection{A form of logical reasoning in policy-based decision making}

The mental state used in the 'policy apply reasoning module' is symbolised and shown in Table 3 . In addition, Table 4 shows the logical formulas that suggest a form of logical reasoning in policy-based decision making. For example, the second line in Table 4 suggests the logical structure of the policy apply reasoning module. In the policy apply reasoning module, $I$ is obtained from $C$ and $P o$, which satisfies ' $C \cup P o r I$ ', thus, this module functions to drive deduction.

Table 3. Mental state symbols in deliberation-based decision making

\begin{tabular}{cl}
\hline Symbols of the mental state & \multicolumn{1}{c}{ Explanation } \\
\hline$C$ & Cognitions of surroundings \\
Po & Policy to form a certain intention in the surroundings \\
& which have been experienced \\
$I$ & Intention, Proactive attitude driving future actions \\
$K t$ & Knowledge of triggers required to execute the intention \\
$A$ & Action related to the design, design operation \\
\hline
\end{tabular}

Table 4. A form of logical reasoning in deliberation-based decision making

\begin{tabular}{ccc}
\hline Reasoning module & Condition & A form of logical reasoning \\
\hline Policy reasoning module & $C \cup P o \vdash I$ & Deduction to obtain $I$ from $C$ and $P o$ \\
Execution determining module & $K t \cup I \cup C \vdash A$ & Deduction to obtain $A$ from $K t, I$ and $C$ \\
\hline
\end{tabular}




\subsection{Typology of error in design rationales}

This research has categorised error in design rationales into three main categories. Two categories appear in the decision premise mechanism and the other appears in the decision making mechanism.

This paper defines 'premise wrongness' and 'premise insufficiency' as error in the decision premise mechanism. 'Premise wrongness' means the fault of the decision premises that referred to the reasoning module in the decision making mechanism. Reasoning is a mental process through which some attitudes of us give rise to a new attitude of us (Broome, 2014). However, if the premise is wrong, the result will also be wrong. Such error is categorised in this type. 'Premise insufficiency' means a lack of decision premises that should be referred to the reasoning module. Even when the premises of reasoning in the design are insufficient, the conclusions can cause errors, such as oversights of decision criteria (Ullman, 2001). Such errors caused by imperfection of knowledge belong to this type. Furthermore, this paper defines 'reasoning wrongness' as error in the decision making mechanism. It means the conclusion cannot be obtained logically from the premise. Even if the premise is true, if the reasoning is not valid, the reasoning cannot be sound. This type of error may occur in poor design environments, such as extremely limited time for design, for example, calculation errors related to the material strength of the construction.

As described above, error can occur in both abduction and deduction. Table 5 shows the typology of error in the design rationale.

Table 5. Typology of error in the design rationale

\begin{tabular}{|c|c|c|c|}
\hline & Premise wrongness & Premise insufficiency & Reasoning wrongness \\
\hline $\begin{array}{l}\text { Abduction } \\
\text { error }\end{array}$ & $\begin{array}{l}\text { - Decision premises that } \\
\text { are referred to the } \\
\text { reasoning module are } \\
\text { faulty. }\end{array}$ & $\begin{array}{l}\text { - Decision premises that } \\
\text { are referred to the } \\
\text { reasoning module are } \\
\text { insufficient. }\end{array}$ & $\begin{array}{l}\text { - The conclusion } \\
\text { contradicts the decision } \\
\text { premises. }\end{array}$ \\
\hline $\begin{array}{l}\text { Deduction } \\
\text { error }\end{array}$ & $\begin{array}{l}\text { - Decision premises that } \\
\text { are referred to in the } \\
\text { reasoning module are } \\
\text { faulty. }\end{array}$ & $\begin{array}{l}\text { - Decision premises that } \\
\text { are referred to in the } \\
\text { reasoning module are } \\
\text { insufficient. }\end{array}$ & $\begin{array}{l}\text { - The conclusion cannot } \\
\text { be obtained from the } \\
\text { decision premises } \\
\text { logically. }\end{array}$ \\
\hline
\end{tabular}

\section{APPLICATION}

\subsection{Setting}

In this paper, the case of the collapse of the Tacoma Narrows Bridge, which is a suspension-type bridge is used to analyse the errors of its design rationale. This bridge was constructed in the USA in the state of Washington in 1940, but it collapsed only 4 months after its completion. This bridge was designed based on 'deflection theory', which states that the force applied to the suspension bridge girder becomes relatively smaller as the weight of the cable becomes heavier, and as the deflection of the suspension bridge floor under load increases. Inspired by this theory, people during this era, including the designer, thought that a suspension bridge with a long span could be constructed successfully based on an easily bending and light bridge floor. However, on a windy day with a crosswind blowing at 19 meters per second, a deflection occurred which caused a self-excited vibration. As a result, the suspension bridge collapsed.

The failure knowledge database provided by the association for the study of failure reported the following two factors as causing bridge collapses (Nakao, 1996).

- The exceptional flexibility and small resistance against twisting of the bridge allowed it to pick up the oscillation quickly.

- The shape was aerodynamically unstable. The H-shaped girders allowed the air flow to easily separate at the edges, and the vortex generation happened to match the oscillation of the girders. The wind-generated vortices moved the girders that then generated new vortices. The designers were unaware of this mechanism of wind excited vibration.

These factors are from a mechanical point of view. In contrast, this study is analysed from the design rationale point of view, using the model proposed in this paper. The analysis in this study will refer to the data provided by the association for the study of failure (Nakao, 1996). This data includes information about the background of the accident, the event and course about accident, the cause of 
the accident, the immediate action and the current status of the Tacoma Bridge, organised to generate knowledge based on the failure. In particular, the background of the accident in this data includes information about the designer's decision making. This study structures this information in terms of the proposed model and analyses the errors of the design rationale.

This case application particularly focuses on the decision making process in which the designer adopted the $\mathrm{H}$-shaped flat bridge girder, which was regarded as a failure factor especially from a view point of mechanics, and identifies the errors in the design rationale. Although the fact whether the designer reasoned through deliberative-based or policy-based process cannot be read from this data, this study assumes that he reasoned through deliberative-based process, which is more thoughtful process than policy-based process. Missing information, which is not available in the data, was assumed by the researchers.

\subsection{Application result}

As shown in Table 6, the researchers assumed that the decision making of the designer was based on deliberation based decision making and captured the mental state components related to decision making. For example, the component of action option $O p$ is $o p 1$ and $o p 2$, namely, ' $O p=\{o p 1, o p 2\}$ '. In Table 6, Ob, Km, Cp, Kf, Ct and $K t$ are the mental states of the decision premises, which refer to decision making. On the other hand, $O p$ and $I$ are mental states of the decision making result. Figure 2 shows the logic in the process of the designer's reasoning. In this way, design rationale is captured and formalised in this case based on the proposed model.

Moreover, errors in the captured design rationale were identified. In this case, the designer used a means-end reasoning module, filtering module, and execution determining module. However, because the mental states referred to in the execution determining module could not be captured, the meansend reasoning module and filtering module were analysed.

Table 6 Components of designer's mental state

\begin{tabular}{|c|c|c|}
\hline $\begin{array}{l}\text { Symbols of } \\
\text { mental state }\end{array}$ & Components & Explanation \\
\hline $\mathrm{Ob}$ & $o b$ & Constructing the long span suspension bridge in low cost \\
\hline$K m$ & $k m$ & $\begin{array}{l}\text { The suspension bridge with a long span can be constructed } \\
\text { successfully based on the bridge floor easy to bend and light }\end{array}$ \\
\hline \multirow[t]{2}{*}{$O p$} & opl 1 & Designing an $\mathrm{H}$-shaped flat bridge girder \\
\hline & op2 & Do not using truss structure for bridge girder \\
\hline \multirow[t]{2}{*}{$C p$} & cpl 1 & Material cost for bridge can be economised \\
\hline & cp2 2 & The rigidity against static load is sufficient \\
\hline \multirow[t]{2}{*}{$K f$} & $k f 1$ & High cost \\
\hline & $k f 2$ & A lack of rigidity against the static load \\
\hline \multirow[t]{2}{*}{$I$} & $i 1$ & Designing an $\mathrm{H}$-shaped flat bridge girder \\
\hline & $i 2$ & Do not using truss structure for bridge girder \\
\hline$C t$ & $c t$ & Unknown \\
\hline$K t$ & $k t$ & Unknown \\
\hline \multirow[t]{2}{*}{$A$} & $a 1$ & Designing an $\mathrm{H}$-shaped flat bridge girder \\
\hline & $a 2$ & Do not using truss structure for bridge girder \\
\hline
\end{tabular}

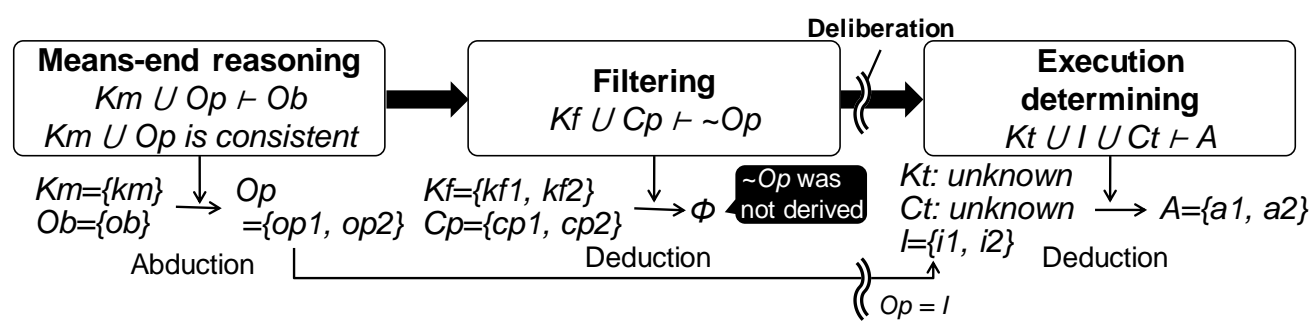

Figure 2. The process of the designer's reasoning 


\subsubsection{The error in the means-end reasoning module}

The mental states of the decision premise that were referred to in means-end reasoning module are $\mathrm{Ob}$ and $\mathrm{Km} . \mathrm{Ob}$ is true but $\mathrm{Km}$ is obviously false because the suspension bridge constructed based on $\mathrm{Km}$ obviously does not have enough wind resistance stability and cannot withstand strong winds. Hence, means-end reasoning module includes premise wrongness. Meanwhile, $\mathrm{Ob}$ can be obtained from $\mathrm{Km}$ and $O p$, which is consistent with decision premise of designer. Hence, this reasoning module, which obtained $O p$ from $O b$ and $\mathrm{Km}$ through abduction, does not include reasoning wrongness.

\subsubsection{The error in the filtering module}

The mental states of the decision premise that were referred to in means-end reasoning module are $C p$ and $K f$. Both are true but $K f$, whose components are $k f l$ and $k f 2$ is not sufficient. It lacks the perspective of wind resistant stability. Therefore, the decision premises that were referred to by this module are insufficient. On the other hand, ' $O b=\{o p 1, o p 2\}$ ' was not rejected in this filtering module. This is valid because both $\sim o p 1$ and $\sim o p 2$ are not obtained from $C p$ and $K f$. Therefore, this filtering module does not include reasoning wrongness.

\subsubsection{The result of design rationale analysis}

As mentioned, the errors in the design rationale, in this case, are identified (a) premise wrongness in abduction, which was included in the means-end reasoning module, and (b) premise insufficiency in deduction, which was included in the filtering module. As these errors in design rationale occurred at the same time, the action which reduced robustness of the suspension bridge was executed finally.

The design rationale error (b) in this case means $K f$ is not sufficient because it lacks a wind resistant stability perspective. The cause of this is that the response of the bridge to the wind was unknown at the time of designing the bridge. Thus, it was extremely difficult to prevent such error. On the other hand, the design rationale error (a) in this case means the wrongness of $\mathrm{Km}$. Km, which is knowledge of means-end to achieve design objective, was probably formalised from 'deflection theory'. This theory does not state the means to construct the suspension bridge directly, but the designer formalised the $K m$, which is the knowledge to construct the suspend bridge, only based on an interpretation of this theory. This was a serious mistake on the part of the designer, and as a result, the bridge could not withstand strong winds and collapsed.

\section{DISCUSSINON AND CONCLUSION}

In the case study, the combination of design rationale error (a) and (b) finally caused the bridge to collapse. Specifically, (b) was difficult to prevent because the wind pressure characteristics for $K f$ were unknown during that era. However, even if there was insufficient knowledge such as (b), it is highly assumed that an incorrect design process could be avoided by preventing (a). The reason why (a) occurred is that the designer formed $K m$ by expanding and interpreting deflection theory in the context of structural mechanics. This result can be generalised to suggest that 'forming $\mathrm{Km}$ only based on single domain knowledge can lead to error in means-end reasoning'. Designers will be able to prevent similar design rationale error from occurring by referring to this knowledge. In this way, it is possible to accumulate effective knowledge for designers to make decisions by establishing an analysis method of design rationale error. It is also expected that accumulated design knowledge can be utilised to practically educate designers. The proposed method can contribute to robust artifact design. The contribution to practitioners is that, even if a design fails, case analysis and knowledge generation based on this method can draw out the potential utilisation of the design rationale in failed cases, thereby increasing the robustness of future design. Rather than simply identifying inconsistencies and contradictions in the design rationale, the paper suggests that a fundamental understanding of why designers make such errors should be developed.

In addition, the study suggests that the interpretation of captured design rationales may differ depending on what cognitive nature the analyst assumes the designer as a subject. Since the analyst cannot directly observe the designer's reasoning process, he/she can only infer it from the designer's actions, his discourses and his surroundings. Thus, to capture and interpret the design rationale, it is necessary to know what kind of cognitive nature the designer has. The study referred the theory of intention to model how designers make rational decisions with limited cognitive resources, and then shows the typology of errors that can still occur. This provides a perspective to obtain and analyse the 
design rationale and enables errors to be identified, thereby demonstrating the potential of knowledge generation for robust design. The point of the contribution to design researchers is that this study indicates the requirement for design rationale researchers to clarify what assumptions they stand for about the designer's cognitive characteristics.

While this study constructed a mental model of designer based on Bratman's theory and corresponded each reasoning module to deduction or abduction, only the module of deliberation was excluded from the scope of this study. This reasoning is not always based on logical form or objective evidences, but may be based on the designer's personal judgements of value. To formalise the reasoning rules in this reasoning module needs to be based on the hypothesis of how the designer judges value, as is the case with utility functions in economics, for example. Whereas this paper proposed a method for analysing errors in design rationale, it was found throughout the application that other types of error caused in the decision premise mechanism. Future work must also include a refinement of the logical structure in the decision premise mechanism and the deliberation reasoning module.

\section{REFERENCES}

Arora, V., Greer, E.J. and Tremblay, P. (1992), “A framework for capturing design rationale using granularity hierarchies", Proceedings of the Fifth International Workshop on Computer-Aided Software Engineering, pp. 246-247.

Bracewell, R., Wallace, K., Moss, M. and Knott, D. (2009), "Capturing design rationale”, Computer-Aided Design, Vol. 41 No. 3, pp. 173-186.

Bratman, M.E., Israel, D.J. and Pollack, M.E. (1988), "Plans and resource-bounded practical reasoning", Computational Intelligence, John Wiley \& Sons, Ltd, Vol. 4 No. 3, pp. 349-355.

Broome, J. (2014), "Normativity in reasoning", Pacific Philosophical Quarterly, John Wiley \& Sons, Ltd, Vol. 95 No. 4, pp. 622-633.

Domeshek, E.A. and Holman, E. (2002), “Web-based design coordination”, International Design Engineering Technical Conferences and Computers and Information in Engineering Conference, Vol. 36215, pp. 167-176.

Garcia, A.C.B. and Howard, H.C. (1992), “Acquiring design knowledge through design decision justification”, Artificial Intelligence for Engineering Design, Analysis and Manufacturing, Cambridge University Press, Vol. 6 No. 1, pp. 59-71.

Hansen, C. and Andreasen, M.M. (2004), “A mapping of design decision-making”.

Hansen, C.T. and Andreasen, M.M. (2000), "Basic thinking patterns of decision-making in engineering design", in Meerkamm, H. (Ed.), MCE 2000 - International Workshop on Multi-Criteria Evaluation, pp. 1-8.

Kunz, W. and Rittel, H.W. (1970), Issues as Elements of Information Systems, Working Paper, Center for Planning and Development Research.

Lakin, F., Wambaugh, J., Leifer, L., Cannon, D. and Sivard, C. (1989), “The electronic design notebook: Performing medium and processing medium", The Visual Computer, Vol. 5 No. 4, pp. 214-226.

Lee, J. (1997), "Design rationale systems: Understanding the issues”, IEEE Expert, Vol. 12 No. 3, pp. $78-85$.

Lee, J. and Lai, K.-Y. (1991), "What's in design rationale?", Human-Computer Interaction, Taylor \& Francis, Vol. 6 No. 3-4, pp. 251-280.

Nakao, M. (1996), "Collapse of Tacoma Narrows Bridge”, University of Tokyo, Tokyo.

Pokojski, J., Oleksiński, K. and Pruszyński, J. (2019), “Conceptual and detailed design knowledge management in customized production - Industrial perspective", Journal of Computational Design and Engineering, Warsaw University of Technology, Faculty of Automotive and Construction Machinery Engineering, Institute of Machine Design Fundamentals, Narbutta 84, Warsaw, Poland, Vol. 6 No. 4, pp. 479-506.

Shipman, F.M. and McCall, R.J. (1997), "Integrating different perspectives on design rationale: Supporting the emergence of design rationale from design communication", Artificial Intelligence for Engineering Design, Analysis and Manufacturing, Cambridge University Press, Vol. 11 No. 2, pp. 141-154.

Simon, H.A. (1947), Administrative Behavior, Simon and Schuster.

Takeda, H., Veerkamp, P. and Yoshikawa, H. (1990), "Modeling design process”, AI Magazine, Vol. 11 No. 4 SE-Articles, p. 37.

Ullman, D.G. (2001), "Robust decision-making for engineering design”, Journal of Engineering Design, Taylor \& Francis, Vol. 12 No. 1, pp. 3-13.

Wang, H., Johnson, A.L. and Bracewell, R.H. (2012), "The retrieval of structured design rationale for the re-use of design knowledge with an integrated representation”, Advanced Engineering Informatics, Vol. 26 No. 2 , pp. 251-266. 